\title{
Is there value of tumor stromal infiltrating lymphocytes for response assessment to chemoradiation in esophageal squamous cell carcinoma?
}

\author{
Xin Wang ${ }^{1}$, Wang Jing ${ }^{2}$, Steven H. Lin ${ }^{3}$ \\ ${ }^{1}$ Department of Radiation Oncology, Tianjin Medical University Cancer Institute and Hospital, Tianjin Medical University, Tianjin 300000, China; \\ ${ }^{2}$ Department of Radiation Oncology, Shandong Cancer Institute, Jinan 250000, China; ${ }^{3}$ Department of Radiation Oncology, The University of Texas \\ MD Anderson Cancer Center, Houston, Texas, USA \\ Correspondence to: Steven H. Lin, MD, PhD. Department of Radiation Oncology, The University of Texas MD Anderson Cancer Center, Houston, \\ Texas 77030, USA. Email: shlin@mdanderson.org. \\ Provenance: This is an invited article commissioned by the Section Editor Dr. Hsin-Hua Nien (Attending Physician, Department of Radiation \\ Oncology, Cathay General Hospital, Taipei). \\ Comment on: Qian D, Wang Y, Zhao G, et al. Tumor Remission and Tumor-Infiltrating Lymphocytes During Chemoradiation Therapy: Predictive \\ and Prognostic Markers in Locally Advanced Esophageal Squamous Cell Carcinoma. Int J Radiat Oncol Biol Phys 2019;105:319-28.
}

Submitted Nov 01, 2019. Accepted for publication Nov 13, 2019.

doi: $10.21037 / \mathrm{atm} .2019 .11 .82$

View this article at: http://dx.doi.org/10.21037/atm.2019.11.82

Although esophagectomy as a single modality remains the cornerstone of treatment for resectable esophageal carcinoma, the incorporation of chemo-radiotherapy (CRT) as neoadjuvant therapy improves overall survival and R0 resection rate compared to surgery alone. Both the CROSS and the NEOCRTEC 5010 trials have demonstrated that neoadjuvant CRT (nCRT) significantly improves overall survival compared to surgery alone, in both adenocarcinoma and squamous cell carcinoma (1-3), without contributing to the morbidity of surgery. Although surgery is still needed after CRT to render the best cure rate, about $13 \%$ to $40 \%$ of EC patients treated with nCRT will achieve a pathologic complete response ( $\mathrm{pCR}$ ), presenting favorable long-term outcomes compared with patients without pCR $(1,2,4)$. For patients with a complete response after CRT, there is no consensus on the best treatment approach. Meta-analysis showed no significant difference in long-term survival between CRT and surgery and definitive CRT $(5,6)$. It is therefore reasonable to proceed with active surveillance without surgery for these patients, in order to avoid the potential morbidity and mortality of surgery, if there was a way to identify these patients early on. However, being able to accurately identify tumors that will sustain complete response to induction therapy is still an area of unmet need (7). Using clinical parameters, such as magnetic resonance imaging (MRI), computed tomography (CT) and PET/CT, have failed to accurately predict $\mathrm{PCR}$ after nCRT (8-10). Diagnostic methods to evaluate clinical CR (cCR) are also not useful since about $19-26 \%$ patients with cCR failed to reach pCR after surgery. Recent attempts to use endoscopic ultrasonography with bite-on-bite biopsies to identify residual islands of cancer cells appears to yield early promising results, by missing only $10 \%$ residual disease (7), this is still a highly experimental and yet to be fully proven in an ongoing randomized trial. There is still a need to identify biomarkers that could even more accurately identify tumors that will sustain pCR after nCRT.

Given the importance of the immune system in cancer therapy response and in disease surveillance, some studies have revealed that tumor-infiltrating lymphocytes (TILs) correlated with response of neoadjuvant therapy and improved survival $(11,12)$. TILs are located in intratumoral and stromal compartments, and composed of various lymphocytes with diverse activities, including $\mathrm{CD}^{+}$, $\mathrm{CD}^{+}, \mathrm{Foxp}^{+}$, and $\mathrm{CD} 57^{+}(13,14)$. Emerging evidence suggests that the amount of lymphocyte infiltration of primary tumors predicts favorable survival in a number of tumor types $(15,16)$. Qian et al. successfully built a model combining tumor regression grade and TILs in locally advanced esophageal squamous cell carcinoma (LA-ESCC) 
from post-CRT biopsy to predict the treatment response of neo-CRT and definitive CRT. When response was evaluated by biopsies alone, the sensitive and specificity were only $66.7 \%$ and $55.4 \%$. After adding the TIL factor into the evaluation methods, the sensitivity and specificity of predicting pCR greatly increased to $86.7 \%$ and $90.0 \%$, respectively (17).

Using TILs as a surrogate biomarker, the Qian et al.'s study pointed to the importance of measuring the magnitude of the immune response to the tumor from CRT as a potential to identify pCR. However, there are still some contentious areas related to this area of research. Their study suggested that TILs within the tumor stroma was an important factor, while Jiang et al. suggested that TILs associated with tumor cells were associated with better survival (13). Although several studies have already demonstrated that TILs in general were associated with better outcomes, some have shown that TIL subsets, especially $\mathrm{CD}^{+} \mathrm{T}$ cells, were related to survival $(18,19)$. As to the level of TILs that would be prognostic, there is also no consistent cutoff value that exists. Some used a $10 \%$ cutoff (13), while others used either $20 \%$ or $50 \%$ $(20,21)$, and in Qian et al.'s study, 60\% was adopted. There is substantial heterogeneity between studies that could account such differences. Many used pretreatment tumor biopsies, while others used samples at the completion of CRT (17), or from the surgical specimen (13). The samples will of course vary based on the depth of the biopsies, as well as the operators' experience. Without adequately correcting for sampling heterogeneity or normalizing the proportion of TILs in either tumor or stroma calls into question the use of TILs as a reliable biomarker.

Other limitations including the relatively small sample size and the short follow-up time to reliably score overall survival. It is also quite clear that determining the histomorphologic regression of tumor tissue after nCRT is also subject to pathologic interpretation and has quite a bit of interobserver variability among pathologists. Moreover, there are differences between tumor regression at the primary site and at the metastatic lymph nodes, and both could differentially influence survival (22). However, Qian et al. prioritized the response rate and lymphocytes infiltration only within the primary tumor site, without taking into account the remission within metastatic lymph nodes.

In summary, Qian et al.'s study provided a potentially promising approach to identify patients whose tumors could be highly sensitive to CRT which confers better survival. Validations using larger datasets from prospective multicenter studies are needed. Accurate and reproducible tools to predict pCR and long-term survival after CRT are still in an urgent need. This is a critical time where these surrogate markers of immunologic activity could be especially useful as a predictive marker in the setting of incorporating immunotherapies with or after CRT.

\section{Acknowledgments}

None.

\section{Footnote}

Conflicts of Interest: The authors have no conflicts of interest to declare.

Ethical Statement: The authors are accountable for all aspects of the work in ensuring that questions related to the accuracy or integrity of any part of the work are appropriately investigated and resolved.

\section{References}

1. van Hagen P, Hulshof MC, van Lanschot JJ, et al. Preoperative chemoradiotherapy for esophageal or junctional cancer. N Engl J Med 2012;366:2074-84.

2. Yang H, Liu H, Chen Y, et al. Neoadjuvant Chemoradiotherapy Followed by Surgery Versus Surgery Alone for Locally Advanced Squamous Cell Carcinoma of the Esophagus (NEOCRTEC5010): A Phase III Multicenter, Randomized, Open-Label Clinical Trial. J Clin Oncol 2018;36:2796-803.

3. Sjoquist KM, Burmeister BH, Smithers BM, et al. Survival after neoadjuvant chemotherapy or chemoradiotherapy for resectable oesophageal carcinoma: an updated metaanalysis. Lancet Oncol 2011;12:681-92.

4. Mariette C, Dahan L, Mornex F, et al. Surgery alone versus chemoradiotherapy followed by surgery for stage I and II esophageal cancer: final analysis of randomized controlled phase III trial FFCD 9901. J Clin Oncol 2014;32:2416-22.

5. Wang J, Qin J, Jing S, et al. Clinical complete response after chemoradiotherapy for carcinoma of thoracic esophagus: Is esophagectomy always necessary? A systematic review and meta-analysis. Thorac Cancer 2018;9:1638-47.

6. Rawat S, Kumar G, Kakria A, et al. Chemoradiotherapy 
in the management of locally advanced squamous cell carcinoma esophagus: is surgical resection required? J Gastrointest Cancer 2013;44:277-84.

7. Noordman BJ, Spaander MCW, Valkema R, et al. Detection of residual disease after neoadjuvant chemoradiotherapy for oesophageal cancer (preSANO): a prospective multicentre, diagnostic cohort study. Lancet Oncol 2018;19:965-74.

8. Fang P, Musall BC, Son JB, et al. Multimodal Imaging of Pathologic Response to Chemoradiation in Esophageal Cancer. Int J Radiat Oncol Biol Phys 2018;102:996-1001.

9. Vollenbrock SE, Voncken FEM, van Dieren JM, et al. Diagnostic performance of MRI for assessment of response to neoadjuvant chemoradiotherapy in oesophageal cancer. Br J Surg 2019;106:596-605.

10. Zhang H, Tan S, Chen W, et al. Modeling pathologic response of esophageal cancer to chemoradiation therapy using spatial-temporal 18F-FDG PET features, clinical parameters, and demographics. Int J Radiat Oncol Biol Phys 2014;88:195-203.

11. Luen SJ, Salgado R, Dieci MV, et al. Prognostic implications of residual disease tumor-infiltrating lymphocytes and residual cancer burden in triple-negative breast cancer patients after neoadjuvant chemotherapy. Ann Oncol 2019;30:236-42.

12. Ochi T, Bianchini G, Ando M, et al. Predictive and prognostic value of stromal tumour-infiltrating lymphocytes before and after neoadjuvant therapy in triple negative and HER2-positive breast cancer. Eur J Cancer 2019;118:41-8.

13. Jiang D, Liu Y, Wang H, et al. Tumour infiltrating lymphocytes correlate with improved survival in patients with esophageal squamous cell carcinoma. Sci Rep 2017;7:44823.

14. Noble F, Mellows T, McCormick Matthews LH, et al.

Cite this article as: Wang X, Jing W, Lin SH. Is there value of tumor stromal infiltrating lymphocytes for response assessment to chemoradiation in esophageal squamous cell carcinoma? Ann Transl Med 2019;7(Suppl 8):S283. doi: 10.21037/ atm.2019.11.82
Tumour infiltrating lymphocytes correlate with improved survival in patients with oesophageal adenocarcinoma. Cancer Immunol Immunother 2016;65:651-62.

15. Tian T, Ruan M, Yang W, et al. Evaluation of the prognostic value of tumor-infiltrating lymphocytes in triple-negative breast cancers. Oncotarget 2016;7:44395-405.

16. Liu K, Yang K, Wu B, et al. Tumor-Infiltrating Immune Cells Are Associated With Prognosis of Gastric Cancer. Medicine (Baltimore) 2015;94:e1631.

17. Qian D, Wang Y, Zhao G, et al. Tumor Remission and Tumor-Infiltrating Lymphocytes During Chemoradiation Therapy: Predictive and Prognostic Markers in Locally Advanced Esophageal Squamous Cell Carcinoma. Int J Radiat Oncol Biol Phys 2019.

18. Zheng X, Song X, Shao Y, et al. Prognostic Role of Tumor-Infiltrating Lymphocytes in Esophagus Cancer: a Meta-Analysis. Cell Physiol Biochem 2018;45:720-32.

19. Zingg U, Montani M, Frey DM, et al. Tumour-infiltrating lymphocytes and survival in patients with adenocarcinoma of the oesophagus. Eur J Surg Oncol 2010;36:670-7.

20. Swisher SK, Wu Y, Castaneda CA, et al. Interobserver Agreement Between Pathologists Assessing TumorInfiltrating Lymphocytes (TILs) in Breast Cancer Using Methodology Proposed by the International TILs Working Group. Ann Surg Oncol 2016;23:2242-8.

21. Salgado R, Denkert C, Demaria S, et al. The evaluation of tumor-infiltrating lymphocytes (TILs) in breast cancer: recommendations by an International TILs Working Group 2014. Ann Oncol 2015;26:259-71.

22. Miyata H, Tanaka K, Makino T, et al. The Impact of Pathological Tumor Regression and Nodal Status on Survival and Systemic Disease in Patients Undergoing Neoadjuvant Chemotherapy for Esophageal Squamous Cell Carcinoma. Ann Surg Oncol 2018;25:2409-17. 